\title{
Central Retinal Artery Occlusion and Third Cranial Nerve Palsy Following Nasal Septoplasty
}

\author{
G. Nageswar Rao ${ }^{a}$ Khageswar Rout ${ }^{a}$ Arttatrana Pal $^{\text {b }}$ \\ ${ }^{a}$ Department of Ophthalmology, Kalinga Institute of Medical Sciences, and \\ ${ }^{\mathrm{b}}$ School of Biotechnology, KIIT University, Bhubaneswar, India
}

\section{Key Words}

Central retinal artery occlusion - Third nerve palsy - Septoplasty - Nasal polypectomy · Visual loss

\begin{abstract}
Background: Postoperative vision loss following routine nasal surgery is an extremely rare and devastating complication. We report a case of unilateral blindness due to central retinal artery occlusion associated with third cranial nerve following septoplasty.

Case Report: We report a patient who developed an unusual central retinal artery occlusion with unilateral blindness following nasal surgery under general anesthesia. A 45-year-old man underwent a nasal septal surgery for severe epistaxis. Soon after recovery, the patient noticed loss of vision in his right eye and was unable to lift his upper eyelid. Upon ophthalmic examinations, we determined that he had right-sided third cranial nerve palsy with central retinal artery obstruction and ptosis of right upper eyelid, restriction of ocular movements, and no perception of light in the right eye. Postoperative computerized tomography scan revealed multiple fractures of the left medial orbital wall, including one near the optic canal. Ptosis and ocular defects were recovered partially, but visual loss persisted until the last follow-up.

Conclusion: This paper highlights one case of complete unilateral blindness from direct central retinal artery occlusion associated with third cranial nerve palsy following an apparently uneventful septorhinoplasty. Ophthalmologists and otolaryngologists should therefore be aware of the possible occurrence of such complications.
\end{abstract}




\section{Introduction}

Postoperative visual loss after non-ocular surgery has been reported and attributed to various causes including ischemic optic neuropathy, central or branch retinal artery occlusion, cortical infarction, rarely external ocular injury, hemorrhagic shock, blood dyscrasia, hypotension, hypothermia, coagulopathic disorders, direct trauma, embolism, and prolonged compression of the eyes [1,2]. However, unilateral or bilateral visual loss following routine nasal surgery is an extremely rare and devastating complication [2-4]. In the majority of these cases, visual loss was attributed to retinal embolism following the retrograde flow of an intra-arterial injection of substances into the turbinate [5]. Visual loss with central retinal artery occlusion as a surgical complication is very uncommon and not many cases have been reported in the literature. We present the first case report of an Indian patient who developed an unusual combination of central retinal artery occlusion with unilateral vision loss and third cranial nerve palsy after nasal surgery.

\section{Case Report}

A 45-year-old man was admitted with epistaxis. After the emergency treatment he was thoroughly examined for the cause of epistaxis. As there was marked left ward deflection of the nasal septum, epistaxis was advised. Under general anesthesia, the mucous membrane on both sides of the nasal septum was elevated after infiltrating a mixture of $2 \%$ xylocaine with 2 lacs units of adrenaline locally and parts of both the cartilaginous and bony septum were removed. The surgery lasted 2 hours and 45 minutes. Before the procedure blood pressure was $140 / 60 \mathrm{~mm} \mathrm{Hg}$ and during operation it was maintained at $90 / 60 \mathrm{~mm} \mathrm{Hg}$. The operation was described as routine and uneventful. Immediately after recovery from general anesthesia, the patient complained of visual loss in the right eye and nasal pain. An ophthalmic examination revealed a visual acuity of 20/20 in the left eye, no light perception in the right eye, right palpebral edema, ptosis of the upper eyelid, chemosis, local erythema and no proptosis (fig. 1). The right pupil was dilated and fixed. Intraocular pressure was within the normal range. Anterior segments were normal in both eyes. The funduscopic examination revealed a pale optic disc with an edematous retina, a small hematoma in the medial portion of the inferior eyelid, a central cherry-red spot at the macula, and attenuated arteries ( $\underline{\text { fig. } 2 a}$ ). Fluorescein angiographic examination revealed a delayed central retinal arterial filling and normal cilioretinal arterial filling (fig. 2b). The rest of the ophthalmic examination was normal in both eyes. A diagnosis of central retinal arterial occlusion of the right eye was made. Massage of the right eye was done to dislodge the possible embolus. As there was no improvement in the status of the retina, a computerized tomography (CT) scan of the orbit was asked to look for any iatrogenic injury to the orbital contents. CT scan of the orbit revealed multiple fractures of the right medial orbital wall, including one near the optic canal (fig. 3). Ptosis, the afferent pupillary defect, and ocular movement improved partially over two postoperative days, but despite treatment with intravenous carbonic anhydrase inhibitors and corticosteroids, the patient had optic atrophy on the subsequent follow-up visits (fig. 4).

\section{Discussion}

While visual loss is a known complication of ethmoidectomies, it seems to be very unusual following nasal surgery. Postoperative visual loss after non-ocular surgery is a rare and infrequent but terrible complication depending on the type of surgery. The major three recognized causes of postoperative visual loss are ischemic optic neuropathy, central retinal artery or vein occlusion, and cerebral ischemia $[2,6-8]$. Plate and Asboe [9] reported three patients who had visual loss in one eye after submucous resection of the nasal septum but no sign of other cranial nerve palsies. 
Moreover, they suggested that a high-pressure intra-arterial injection of local anesthetics in combination with adrenalin in the mucosa during surgery could have led to retrograde flow into branches of the ophthalmic artery with subsequent occlusion of the feeding vessels of the optic nerve. Few publications demonstrated similar cases in which the mechanism responsible for visual loss was a central retinal artery occlusion following injection of local anesthetics into the nasal mucosa during elective septorhinoplasty [4, 9]. Wind [6] reported a patient with temporary visual loss after an injection of anesthetics into the nasal mucosa. Direct trauma to the optic nerve is another apparently rare mechanism of visual loss in nasal rhinoseptoplasty. Plate and Asboe [9] reported that total visual loss associated with sixth nerve palsy, blurring of the ethmoidal cells and maxillary sinus on the same side that support the direct trauma was the suspected mechanism. In our present case, computerized tomography scan reported that the direct trauma to the optic nerve after submucous resection of the nasal septum was confirmed. This case shows that direct optic nerve injury can be an isolated complication of an apparently uneventful surgery. Although not observed by the surgeon, the instrument used to fracture the bony part of the septum may have been placed too high and too laterally in the posterior nasal cavity reaching the optic canal area. The potential for such an occurrence is significant since in many cases the anatomy of the posterior and higher portion of the nasal cavity is somewhat distorted due to a preexistent septum abnormality. Central retinal artery occlusion associated with third cranial nerve palsy is an extremely rare entity. To our knowledge this is the first of its kind in the literature. Considering the medical and legal implications they may come to cope with, ophthalmologists and otolaryngologists should be aware of the possible occurrence of such complications.

\section{Disclosure Statement}

The authors declare that they have no competing interests. 

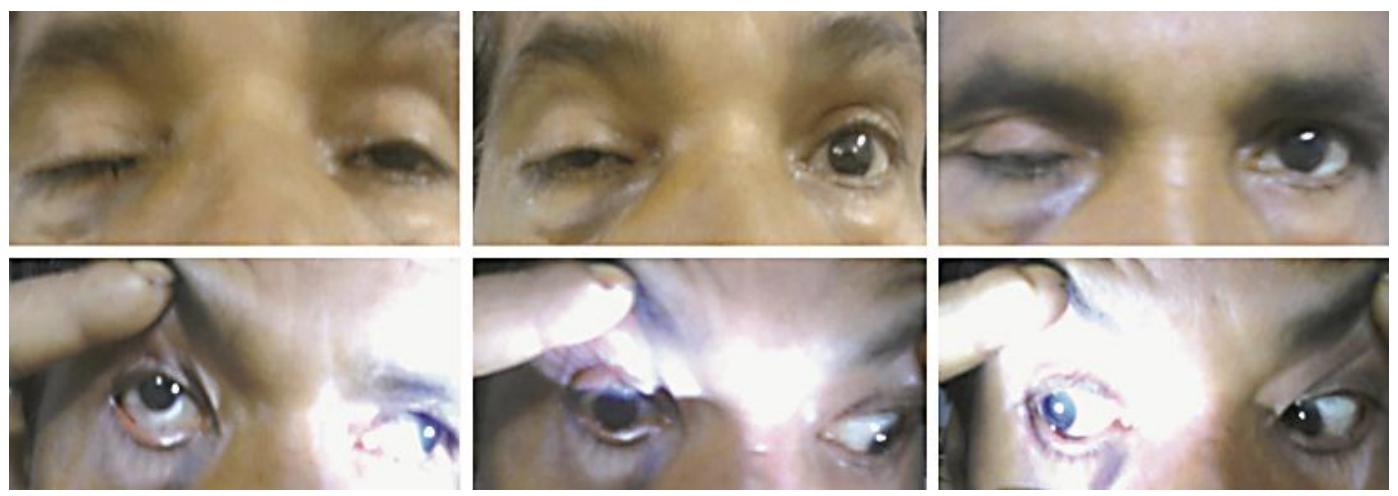

Fig. 1. Patient shows ptosis of right upper eyelid with restriction of adduction, elevation and depression.
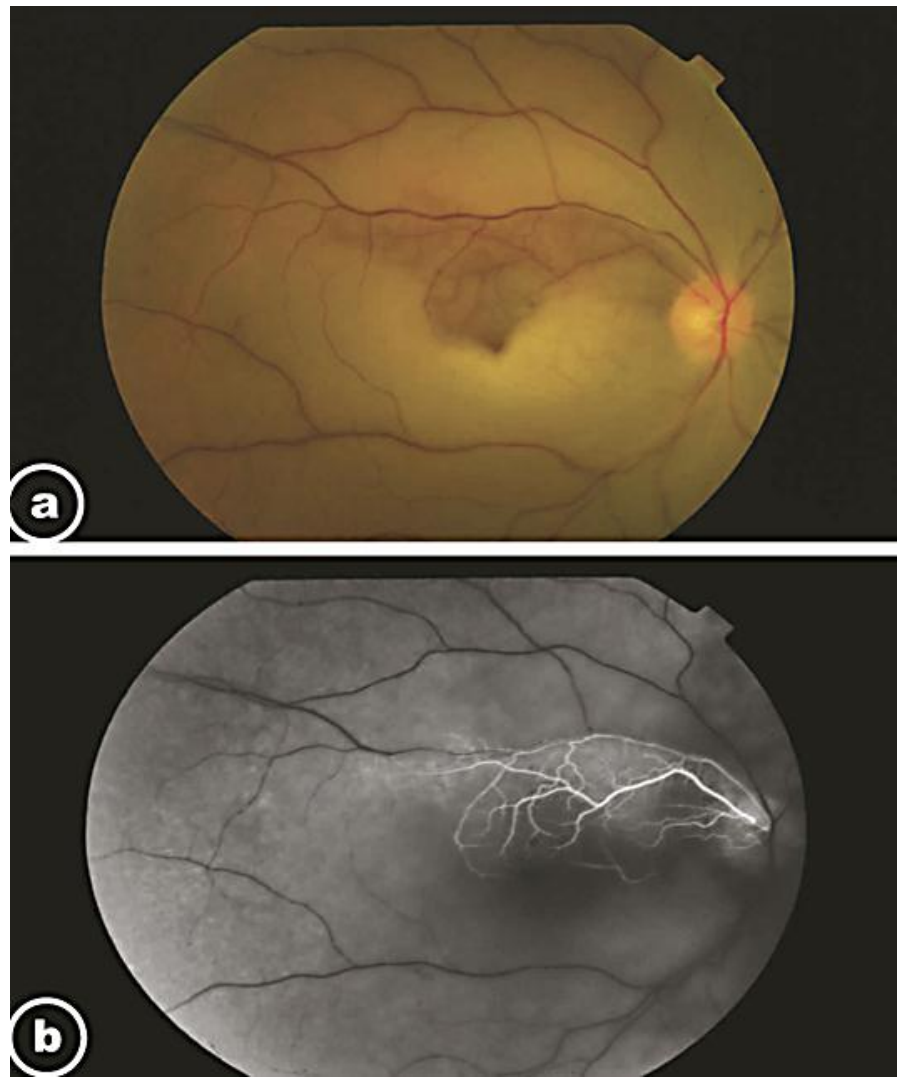

Fig. 2. Color fundus photograph of right eye shows pale retina with cherry red spot (a), and fluorescein angiographic examination shows nonperfusion of central retinal artery with intact cilioretinal artery (b). 


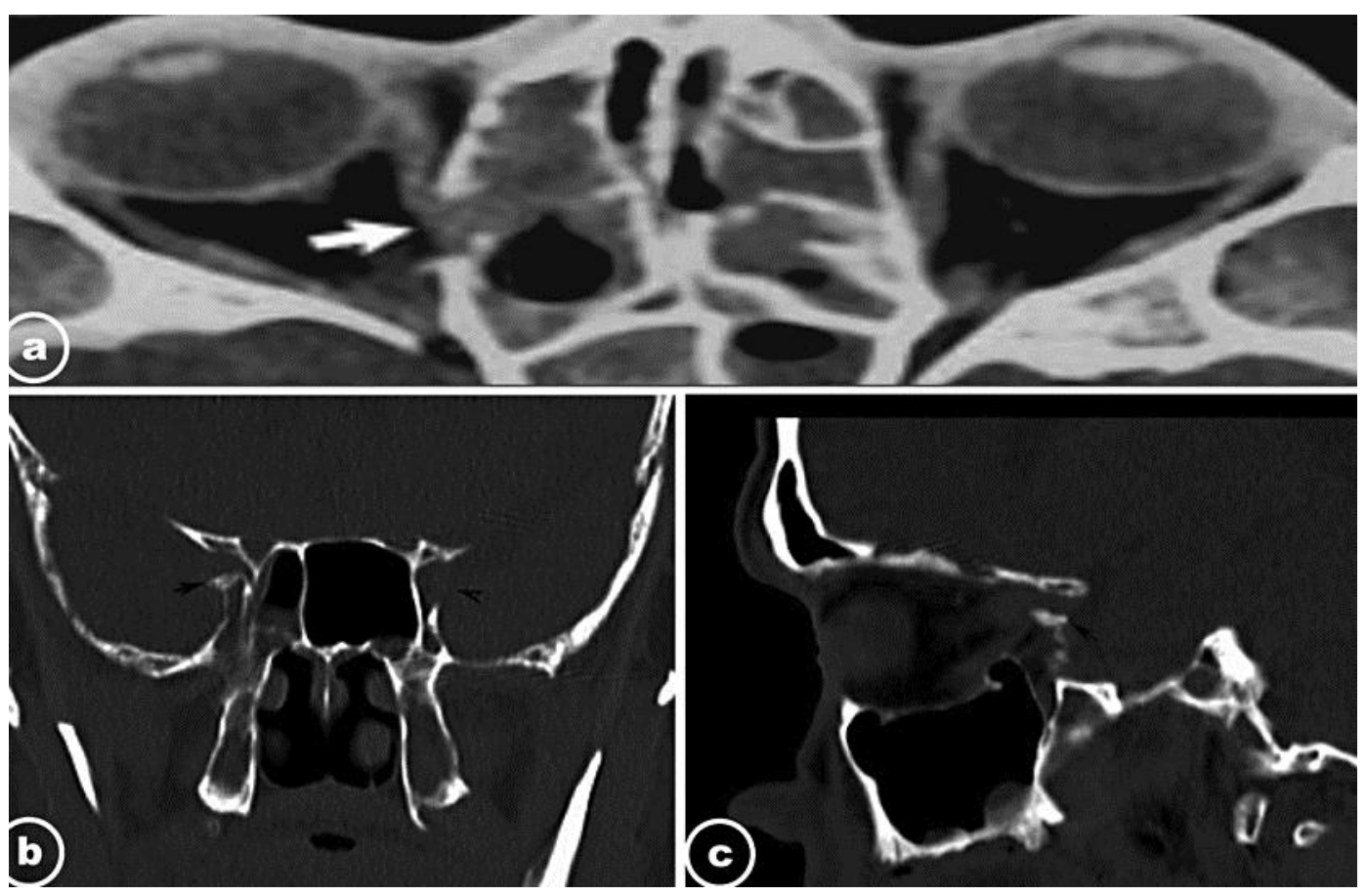

Fig. 3. Computerized tomography scan coronal image showing injured lamina papyracea (arrow). a Axial, b coronal and c sagittal.
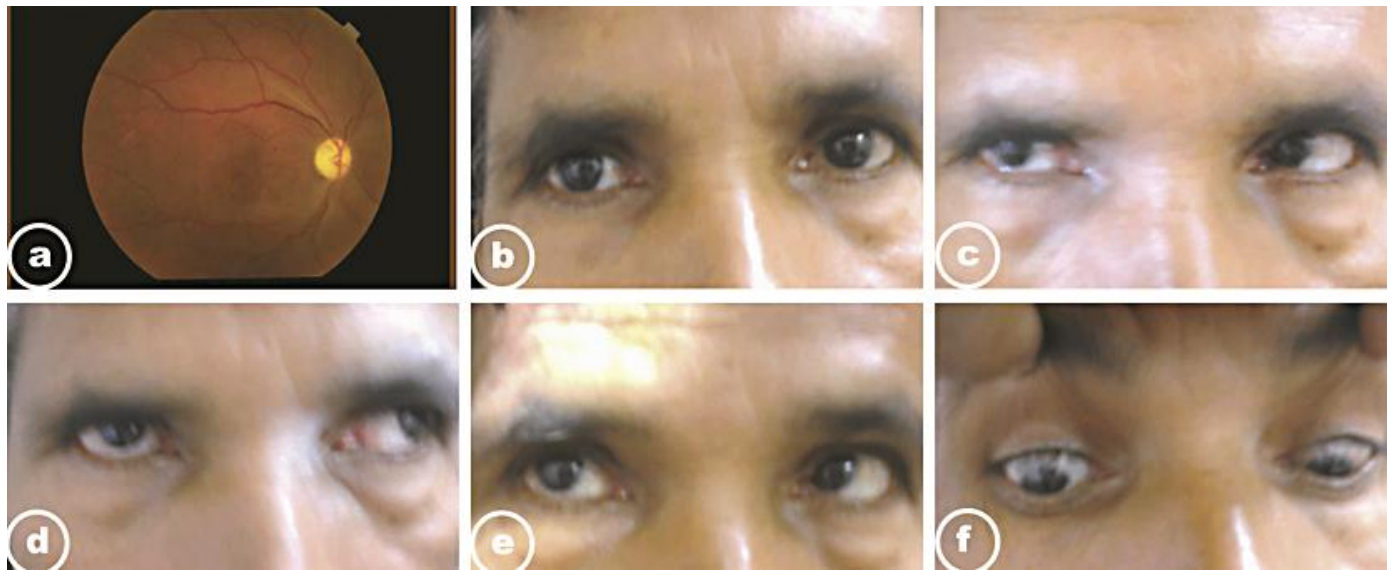

Fig. 4. After three months, fundus photograph of right eye shows pale optic disc (a) and improvement of eye movement and ptotic upper eyelid (b-f). 


\section{References}

1 Stevens WR, Glazer PA, Kelley SD, Lietman TM, Bradford DS: Ophthalmic complications after spinal surgery. Spine 1997;12:1319-1324.

2 Leng T, Moshfeghi DM: Branch retinal artery occlusion after septoplasty. Ophthalmic Surg Lasers Imaging 2010;41:e1-e2.

3 Shihada R, Fradis M, Vardizer Y, Braun J, Luntz M: Unilateral blindness following septoplasty. Ann Otol Rhinol Laryngol 2008;117:528-530.

4 Gomes CC, Sakano E, Lucchezi MC, Panhoca R, Melo RRG, Perda AM: Da visão unilateral pós-septoplastia. Rev Bras Otorrinolaringol 1993;59:142-145.

5 Monteiro ML: Unilateral blindness as a complication of nasal septoplasty: case report. Arq Bras Oftalmol 2006;69:249-250.

-6 Wind J: Blindness as a complication of rhinoplasty. Arch Otolaryngol Head Neck Surg 1988;114:581.

7 Givner I, Jaffe N: Occlusion of the central retinal artery following anesthesia. Arch Ophthalmol 1950;43:197-201.

8 Williams EL, Hart WM Jr, Tempelhoff R: Postoperative ischemic optic neuropathy. Anesth Analg 1995;80:1018-1029.

9 Plate S, Asboe S: Blindness as a complication of rhinosurgery. J Laryngol Otol 1981;95:317-322. 